\title{
Hydrothermally Synthesized Copper Isoleucinate (Bio-MOF-30), Evaluated by Single Crystal XRD Analysis and Drugs Storage Property
}

\author{
Tabinda Sattar ${ }^{* 1}$ and Muhammad Athar \\ ${ }^{1}$ Institute of chemical sciences, B.Z.U Multan, Pakistan
}

Received:01 June, 2017; Accepted: 28 June, 2017; Published: 10 July, 2017

*Corresponding authors: Tabinda Sattar, PhD Scholar, Institute of chemical sciences, B.Z.U Multan, Pakistan. E-mail: tabindasattarahsan22@ gmail.com

\begin{abstract}
TBio-MOF-30 [Cu( $\left.\left.\mathrm{C}_{6} \mathrm{H}_{12} \mathrm{NO}_{2}\right)_{2}\left(\mathrm{H}_{2} \mathrm{O}\right)\right]$ has been hydrothermally synthesized from a biomolecule Isoleucine along with a bio compatible metal cation copper and characterized by single crystal XRD analysis. Bio-MOF-30 crystallized in orthorhombic crystal system with $\mathrm{P} 2{ }_{1} 2_{1} 2_{1}$ space group. In vitro drugs adsorption studies of three drugs i.e. terazosine hydrochloride, telmisartan and glimpiride have been successfully carried out in Bio-MOF-30. HPLC studies of drugs loaded Bio-MOF-30 have been carried out to estimate the amount of adsorbed drugs and also to observe slow release of drugs after intervals. Thermograms and powder XRD patterns of Bio-MOF-30 before and after drug adsorption have been recorded to elaborate the phenomenon of drugs adsorption in Bio-MOF-30.
\end{abstract}

Keywords: Metal Organic Frameworks; Isoleucine; Copper; Single Crystal XRD Analysis; High Performance Liquid Chromatography (HPLC); Terazosine Hydrochloride; Telmisartan; Glimpiride

\section{Introduction}

Metal Organic Frameworks (MOFs) are the materials which are important due to their tuneable structural features and multifunctional properties [1]. The signature property of MOFs is their porosity, due to which these materials have well replaced their last century's counterpart (coordination polymers) [2,3]. Thousands of MOFs have been prepared in a short period of time since their discovery. Yet this field is so diverse that there is still a wide space to synthesize new MOFs for better applications $[4,5]$.

As the MOFs are based on the organic linker molecules and the coordinating metal cations so their synthesis and structural diversity mainly depend upon the selection of linker as well as metal cations [6,7]. One key advantage is that the possible linker molecules for synthesis of MOFs are infinite including phosphonates, sulfonates, polycarboxylates, pyridyl, phenolates and amines etc [8-10]. So MOFs of desired properties can be designed for synthesis by functionalization of MOFs with suitable linkers and metal cations [11-13].

For synthesis of MOFs with better drugs storage properties, nitrogen containing linkers such as amino acids are the best choices which have no toxic effects [14]. Similarly bio compatible metal cations like copper, nickel and manganese are suitable to synthesize MOFs both for in vitro and in vivo drugs adsorption studies [15].

According to some preliminary research reports, amino group containing linker molecules were used for the construction of frameworks suitable for the drugs adsorption [21-23]. MIL-100, MIL-101(Fe), MIL-101(Cr), MIL-53(Fe), MIL-53 (Cr) have been reported with amount of adsorbed drug $1.37,0.35,0.35,0.22$, $0.21 \mathrm{~g} / \mathrm{g}$ and with time release (days) 6, 3, 3, 21, 21 respectively. Also Bio-MOF-1 and Bio-MOF-100 have been reported in recent years with special topology of amino based linker molecules having drugs storage capacity of $0.2 \mathrm{~g} / \mathrm{g}[24,25]$.

The present work is based on the hydrothermal synthesis of a new bio metal organic framework (Bio-MOF-30) in which bio linker isoleucine and copper cation (trace element necessary for human health) have been used. This new Bio-MOF-30 has been designed to be used as a drug carrier. In vitro studies of drug adsorption have been carried out in the present work, which may lead to the in vivo drug adsorption investigations.

\section{Experimental}

All chemicals were purchased from Sigma Aldrich and these were of reagent grade and were used as such.

In a cleaned glass beaker Isoleucine $(0 \bullet 17 \mathrm{~g}, 0 \bullet 2 \mathrm{mmol})$ and copper chloride $\mathrm{CuCl}_{2} \bullet 2 \mathrm{H}_{2} \mathrm{O}(0 \bullet 35 \mathrm{~g}, 0 \bullet 2 \mathrm{mmol})$ were dissolved in $10 \mathrm{~mL}$ distilled water. This solution was well stirred for half an hour. $\mathrm{pH}$ of the solution was maintained at 10 by using sodium carbonate $\left(\mathrm{Na}_{2} \mathrm{CO}_{3}\right)$. This solution was then transferred to a 23 $\mathrm{mL}$ teflon lined autoclave. The autoclave was placed in oven at $150^{\circ} \mathrm{C}$ for three days. After three days fine quality blue colored elongated crystals were obtained from it. These crystals were 
collected, washed with water and acetone and were dried.

Elemental Analysis of Bio-MOF-30, $\left[\mathrm{C}_{12} \mathrm{H}_{26} \mathrm{~N}_{2} \mathrm{O}_{5} \mathrm{Cu}\right]$,

Theoretical: $\mathrm{Cu}=18.59 \%, \mathrm{C}=42.15 \%, \mathrm{H}=7.68 \%, \mathrm{~N}=8.19 \%$,

Experimental: $\mathrm{Cu}=18.67 \%, \mathrm{C}=42.17 \%, \mathrm{H}=7.72 \%, \mathrm{~N}=8.23$ $\%$.

(KBr 4000-400 $\left.\mathrm{cm}^{-1}\right)$ : 3641.4(br), 3188.1(br), 3089.8(br), 2636.5(br), 1633.6(br 1548.7(m), 1416.70(m), 1334.6(m), 1274.9(s), 1218.9(s) 1141.8(s), 1029.9(s), 794.6(s), 511.1(s), $459.0(\mathrm{~s}), 412.7(\mathrm{~s})$.

Thermograms of Bio-MOF-30 before and after drug loading were recorded on SDT Q600 under a $\mathrm{N}_{2}$ atmosphere from $0^{\circ} \mathrm{C}$ to $600^{\circ} \mathrm{C}$ at a heating rate of $10^{\circ} \mathrm{C} / \mathrm{min}$. FT-IR spectra of Bio-MOF-30 (see supplementary material) were obtained from Shimadzu 8400 FT-IR by preparing KBr pellets of Bio-MOF-30. Powder XRD pattern of Bio-MOF-30 before and after drug adsorption were recorded on Bruker $\mathrm{D}_{2}$ Phaser. HPLC studies of the drugs soaked Bio-MOF-30 were carried out on an HPLC instrument, Waters 2695 Separation Module to estimate the amount of drugs adsorbed and also to observe its slow release after intervals.

For the determination of an XRD crystal structure of BioMOF-30, a single crystal was used on a Brucker Smart Apex II CCD area detector diffractometer with graphite monochromated $\operatorname{MoK} \alpha(\lambda=0.71073 \AA$ ) radiation. A crystal of Bio-MOF-30 was mounted in a glass capillary and data were collected at $273 \mathrm{~K}$. For the sake of the data collection and refinements all computer programs used were contained in the Bruker program packages Smart, Saint, and SHELXTL [27].

Direct methods have been used to solve the structure in order to locate the position of copper and most of the hydrogen atoms. Subsequent Fourier Transformation Techniques were used to locate and expand the position of the remaining non hydrogen atoms. Final difference Fourier syntheses described only chemically insignificant electron density. All the hydrogen atoms were placed in surely idealized positions. All non hydrogen atoms were refined by anisotropic method and were further refined with the aid of program SHELXL97 (Sheldrick 1997) by the use of a full- matrix least-square calculation on $\mathrm{F}^{2}$.

\section{Preparation of drug soaked sample of Bio-MOF-30}

In a typical method 0.1 molar solution of the drug (terazosine hydrochloride) was prepared and Bio-MOF-30 was soaked in it. The drug solution was removed after twenty four hours and fresh solution was added to the material. The process was repeated for twenty days on regular basis. The drug soaked material was removed and saved for characterization. TGA and powder XRD studies were carried out for this drug soaked material. Some amount of drug soaked material was placed in distilled water in a vial and aliquot of water containing released drug was separated from vial after one day. Then same amount of fresh water was added to it and after three days aliquot of water containing released drug were separated from it. The same process was repeated after five, seven, nine and eleven days of placing the soaked material in pure water in vial and all these samples were saved for HPLC studies. The whole procedure was also repeated for Bio-MOF-30 by using other drugs (telmisartan, glimpiride).

\section{Results and Discussions \\ Crystallographic studies of Bio-MOF-30}

The new synthesized compound crystallized in orthorhombic crystal system with P212121 space group forming onedimensional crystal lattice based on E-values, successful solution and refinements. SAINT program was used to perform the data reduction. Figure 1 shows the ORTEP drawing of the new material Bio-MOF-30.

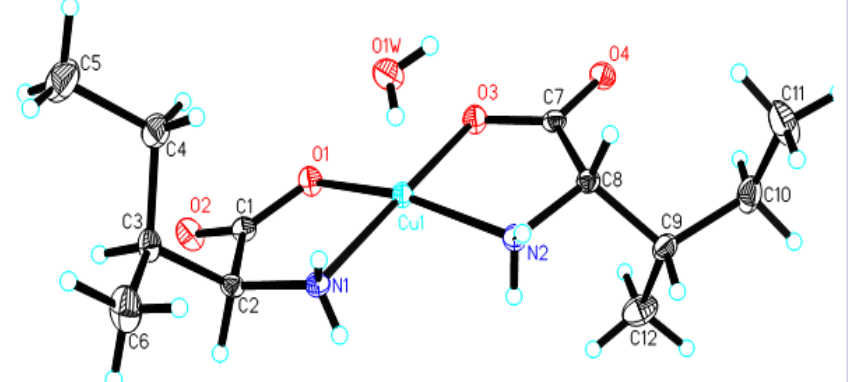

Figure 1: ORTEP drawing of Bio-MOF-30

In an asymmetric unit each copper atom is attached to one water molecule and two bidentate Isoleucinate ligands thus satisfying its five coordination sites. Each of the isoleucinate anion is attached to the copper cation by its nitrogen atom and one of oxygen atoms thus stabilizing its five membered rings. Two isoleucinate ligands coordinate copper in a square plane, satisfying four coordination sites, whereas one water molecule is attached to copper at perpendicular to the isoleucinate-Cu plane. In this way square pyramidal geometry is attained. Different structural views of Bio-MOF-30 along a, b and $c$ axis has been shown below in the Figure 2 .

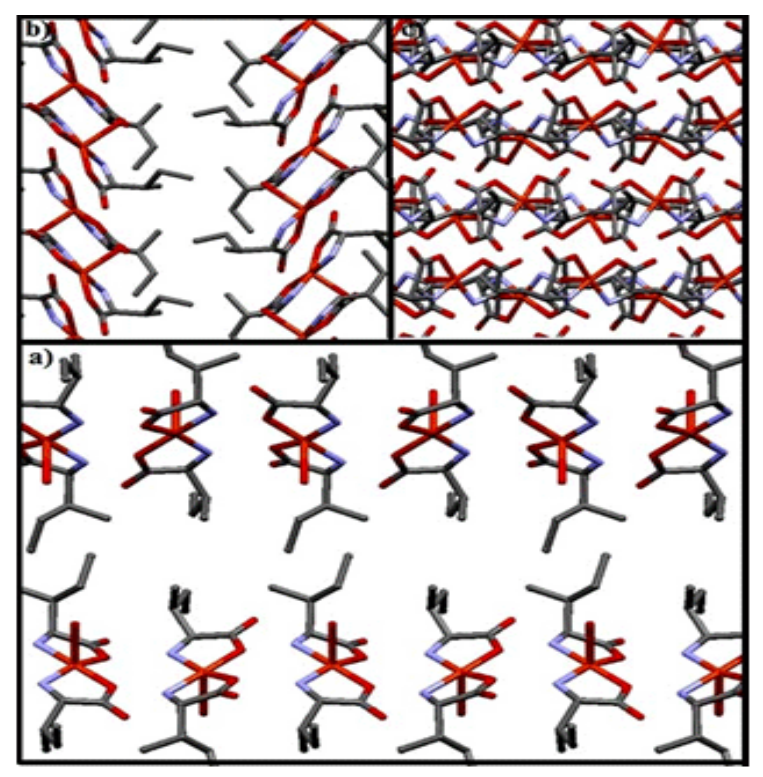

Figure 2: Structural view of Bio-MOF-30 along a, b and $\mathrm{c}$ axis 
The $\mathrm{Cu}-\mathrm{O}$ bond lengths are $1.9504(14) \AA$ for $\mathrm{Cu}(1)-\mathrm{O}(1)$ and $1.9341(14) \AA$ for $\mathrm{Cu}(1)-\mathrm{O}(3)$. Whereas $\mathrm{Cu}-\mathrm{N}$ bond lengths are 1.9947(18) $\AA$ for $\mathrm{Cu}(1)-\mathrm{N}(1)$ and 1.9836(18) $\AA$ for $\mathrm{Cu}(1)-\mathrm{N}(2)$ The value of bond angles of $\mathrm{O}-\mathrm{Cu}-\mathrm{O}$ is $91.96(6)^{\circ}$ for $\mathrm{O}(3)$ $\mathrm{Cu}(1)-\mathrm{O}(1)$ and value of $\mathrm{N}-\mathrm{Cu}-\mathrm{N}$ bond angle is $98.82(8)^{\circ}$ for $\mathrm{N}(2)$ $\mathrm{Cu}(1)-\mathrm{N}(1)$.

One dimensional growth of the crystal lattice has been shown in Figure 2. Certain spaces in the crystal lattice are maintained through hydrogen bonding and this packing through one dimensional pillar of lattice results in the development of pores of discrete sizes which can be used for the storage of drug molecules. CCDC-1417408 contains the supplementary crystallographic data for this paper. The data can be obtained free of charge from the Cambridge crystallographic data centre, 12 Union Road Cambridge CB21EZ, UK (fax: (+44)1223-336-033; or E-mail; deposit@ccdc.cam.ac.uj). The crystal data parameters have been summarized in Table 1 .

Table 1: Crystal data and Structure refinement for BIO-MOF-30

\begin{tabular}{|c|c|}
\hline Empirical formula & $\mathrm{C}_{12} \mathrm{H}_{26} \mathrm{~N}_{2} \mathrm{O}_{5} \mathrm{Cu}$ \\
\hline Formula weight & 341.90 \\
\hline Temperature & $273(2) \mathrm{K}$ \\
\hline Wavelength & $0.71073 \mathrm{~A}$ \\
\hline Crystal system, space group & Orthorhombic, $\mathrm{P} 2_{(1)} 2_{(1)} 2_{(1)}$ \\
\hline Unit cell dimensions & $\begin{array}{c}\mathrm{a}=5 \cdot 0212(7) \AA \\
\mathrm{b}=10 \cdot 4206(14) \AA \\
\mathrm{c}=13 \cdot 0071(16) \AA\end{array}$ \\
\hline Volume & $1564 \cdot 22(12) A^{3}$ \\
\hline Z, Calculated density & $2,1.452 \mathrm{Mg} / \mathrm{m}^{3}$ \\
\hline$F(000)$ & 723 \\
\hline Crystal size & $0.32 \times 0.17 \times 0.11 \mathrm{~mm}$ \\
\hline Theta range for data collection & $2 \cdot 50$ to $25 \cdot 49^{\circ}$ \\
\hline Limiting indices & $\begin{array}{c}-8<=\mathrm{h}<=9,8<=\mathrm{k}<=11, \\
26<=\mathrm{l}<=23\end{array}$ \\
\hline Reflections collected / unique & $9255 / 2901[\mathrm{R}(\mathrm{int})=0 \cdot 0197]$ \\
\hline Completeness to theta $=25.49$ & $99 \cdot 8 \%$ \\
\hline $\begin{array}{l}\text { Maximum and minimum } \\
\text { Transmission }\end{array}$ & 0.8598 and 0.6601 \\
\hline Refinement method & Full-matrix least-squares on $\mathrm{F}^{2}$ \\
\hline Data / restraints / parameters & $2901 / 0$ / 205 \\
\hline Goodness-of-fit on $F^{2}$ & $1 \cdot 062$ \\
\hline Final $R$ indices [I $>2$ sigma(I)] & $\mathrm{R} 1=0.0226, \mathrm{wR} 2=0.0609$ \\
\hline$R$ indices (all data) & $\mathrm{R} 1=0.0243, \mathrm{wR} 2=0 \cdot 0620$ \\
\hline Absolute structure parameter & $0 \cdot 014(13)$ \\
\hline
\end{tabular}

\section{Drugs adsorption in Bio-MOF-30}

Bio-MOF-30 has been explored for its drugs adsorption capacity. Three drugs namely terazosine hydrochloride, telmisartan and glimpiride have been selected for in vitro drugs adsorption studies in Bio-MOF-30. Because these three drugs have applications in treatment of hypertension, high blood pressures, cholesterol and sugar levels in human body respectively so the successful incorporation of these drugs for in vitro studies will lead towards their usage for in vivo studies as well.

\section{Thermogravimetric studies of Bio-MOF-30}

TGA plots of both as synthesized material and drug adsorbed material were recorded as given in Figure 3 below and
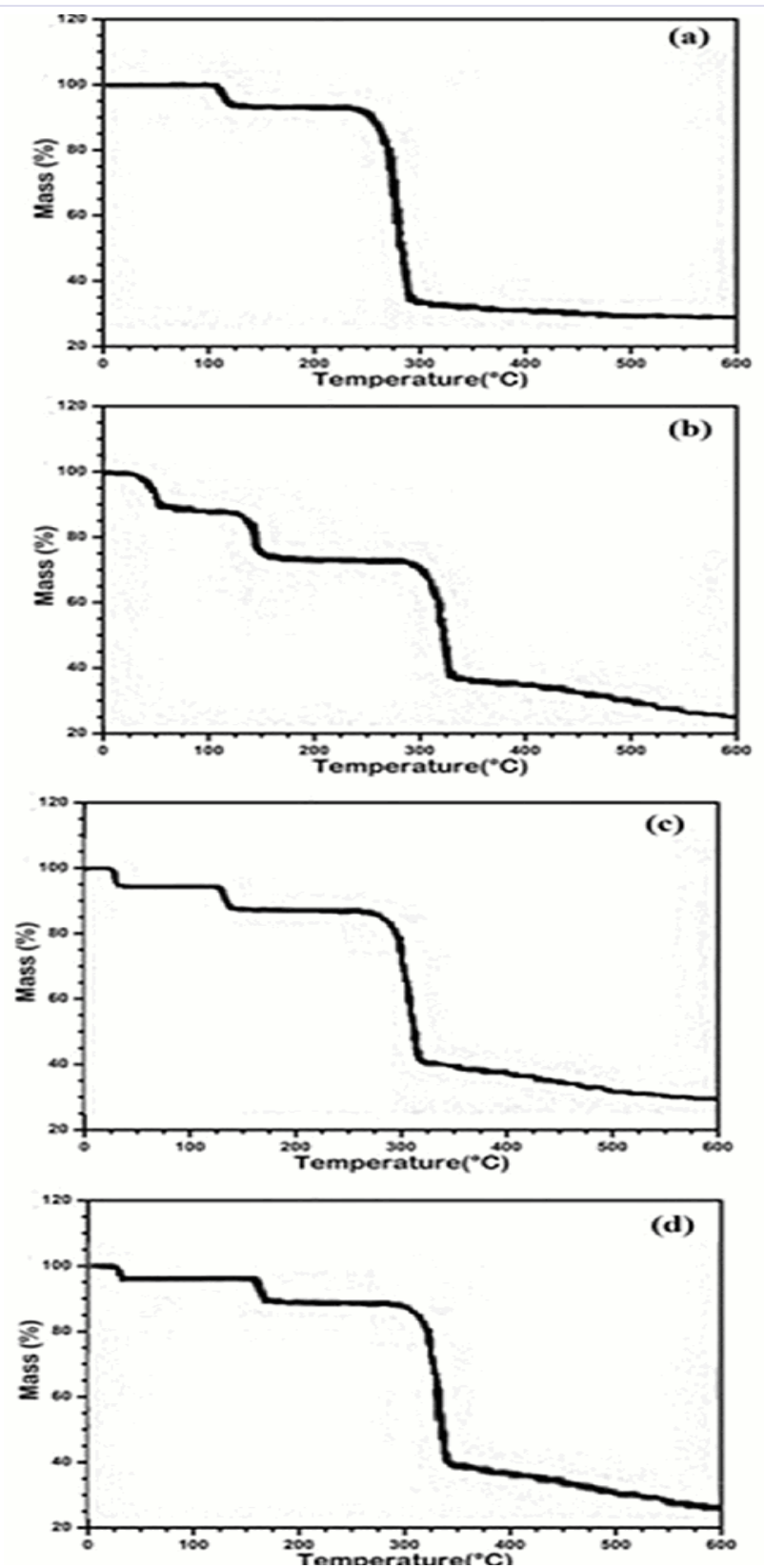

Figure3: Thermogram of Bio-MOF-30 a) Pure b) terazosine loading c) telmisartan loading d) glimpiride loading 
compared for the evidences of drug adsorption in Bio-MOF-30. The thermogram of Bio-MOF-30 before the drugs adsorption shows first weight loss of $6 \%$ at $128^{\circ} \mathrm{C}$ which is due to the loss of coordinated water. Then upto $275^{\circ} \mathrm{C}$ the framework remains intact, after that the decomposition of framework starts along with ligands which continue till $300 \mathrm{oC}$ with major weight loss of $63 \%$. Decomposition continues gradually uptill $600^{\circ} \mathrm{C}$ at which weight loss of $68 \%$ shows complete decomposition of framework and formation of metal oxides.

On the other hand the thermogram of the terazosine loaded Bio-MOF-30 indicates the first weight loss of nearly $11 \%$ at $50^{\circ} \mathrm{C}$ which is due to the loss of adsorbed terazosine molecules. Then up to $145^{\circ} \mathrm{C}$ the framework shows stability after that weight loss of $24 \%$ can be observed up to $166^{\circ} \mathrm{C}$ which is due to removal of coordinated water molecule. Gradual decomposition of framework and ligands starts after $300^{\circ} \mathrm{C}$. After that maximum weight loss of $60 \%$ at $596^{\circ} \mathrm{C}$ shows framework decomposition along with the remaining adsorbed terazosine molecules.

Thermogram of telmisartan loaded Bio-MOF-30 indicates the first weight loss of nearly $5 \%$ at $35 \mathrm{oC}$ which is due to the loss of adsorbed telmisartan molecules. Then up to $150^{\circ} \mathrm{C}$ the framework shows stability, after that a weight loss of $13 \%$ is observed up to $155^{\circ} \mathrm{C}$ which is due to the loss of coordinated water molecule. Then a gradual decomposition of framework and ligands starts which continues till $334^{\circ} \mathrm{C}$ with maximum weight loss of $68 \%$. After that at $590^{\circ} \mathrm{C}$ whole framework decomposes along with the remaining adsorbed telmisartan molecules.

While thermogram of glimpiride loaded Bio-MOF-30 indicates the first weight loss of nearly $4 \%$ at $45 \mathrm{oC}$ which is due to the loss of adsorbed glimpiride molecules. The framework shows stability for some time and at $180^{\circ} \mathrm{C}$ the weight loss of $10 \%$ is observed which is due to the loss of coordinated water molecule. Then up to $345^{\circ} \mathrm{C}$ the framework remains intact, after that gradual decomposition of framework and ligands starts which continues till $355^{\circ} \mathrm{C}$ with maximum weight loss of $45 \%$. At $598^{\circ} \mathrm{C}$ whole framework decomposes with weight loss of $65 \%$ along with the remaining adsorbed glimpiride molecules.

\section{HPLC studies for the estimation of different drugs in Bio-MOF-30}

HPLC studies of drugs soaked Bio-MOF-30 were carried out by using a C-18 Luna $5 \mu 100 \AA$ (2) Column Phenomenex along with PDA Detector (Waters 2996 photo diode). Size of columns used was $250 \times 4.6 \mathrm{~mm}$, while injection volumes of samples used were $20.0 \mu \mathrm{L}$ with a flow rate of $1.0 \mathrm{~mL} / \mathrm{min}$ and with a run rate of 8.9 minutes approximately at ambient temperature. Run time and retention time of all samples of drugs were 13.00 minutes and 10.5 minutes approximately. Mobile phase used for procainamide hydrochloride was $\mathrm{MeOH}$ : H2O: TEA (Tri Ethyl Amine); Citrate Buffer: ACN (Aceto Nitrile) for terazosine hydrochloride and $\mathrm{MeOH}$ : Citrate Buffer for telmisartan. Whereas Sodium dihydrogen phosphate buffer: ACN (Aceto Nitrile) was used as mobile phase for both glimpiride and rosuvastatin respectively.

582399.66, 23276347, 10678092 were mean peak areas calculated for standards of terazosine hydrochloride, telmisartan and glimpiride respectively. Chromatograms, peak areas of all the samples of water containing released drugs (terazosine hydrochloride, telmisartan, glimpiride) collected after different time intervals for HPLC studies for estimation of drug contents, calculations and estimations of different drugs in Bio-MOF-30, have been given as supplementary material.

In Bio-MOF-30 three different drugs (terazosine hydrochloride, telmisartan and glimpiride) have been successfully adsorbed. $0.21 \mathrm{~g} / \mathrm{g}$ material of terazosine hydrochloride has been estimated in Bio-MOF-30. Highest release of terazosine contents from drug adsorbed Bio-MOF-30 was estimated after one day, which gradually decreased with passage of time as has been determined from HPLC calculation of samples collected at different times (see supplementary material) but no terazosine contents were found in aliquot of Bio-MOF-30 taken on the eleventh day.

$0.1 \mathrm{~g} / \mathrm{g}$ material of telmisartan has been estimated in BioMOF-30. Highest release of telmisartan contents were estimated in aliquot of water containing released drug after one day which decreased on the third day and no telmisartan contents were found in aliquot of water containing released drug taken on fifth day.

$0.6 \mathrm{~g} / \mathrm{g}$ material of glimpiride has been estimated in BioMOF-30. Highest release of glimpiride contents were estimated in aliquot of water containing released drug after one day. Next release was observed after three days. But no glimpiride contents were found after three days.

\section{Powder XRD patterns of Bio-MOF-30}

Powder XRD pattern of Bio-MOF-30 in pure form and after drugs adsorption has been recorded as has been given below in Figure 4. Permanent crystalline integrity of Bio-MOF-30 has been revealed from these PXRD pattern after drugs adsorption which indicate that this material has retained its crystallinity even after soaking it in water for several days.
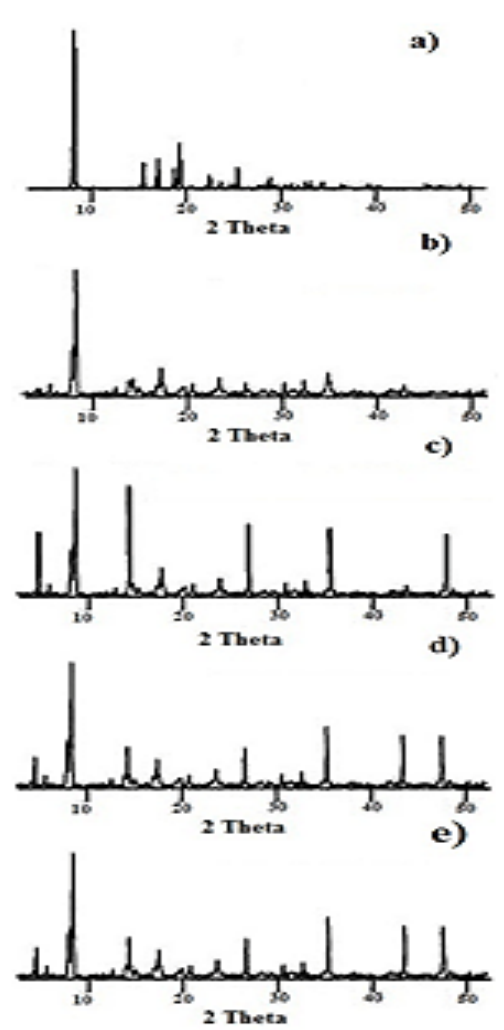

Figure 4: PXRD patterns of Bio-MOF-30 a) simulate b) pure c) after terazosine d) after telmisartan e) after glimpiride 


\section{Conclusion}

In conclusion the present work describes the hydrothermal synthesis of bio-MOF-30 from a bio linker molecule (Isoleucine) and biocompatible metal cation (copper). This work can lead towards the use of biomolecules for construction of frameworks potentially useful for drugs adsorption. Successful in vitro adsorption of three drugs (terazosine hydrochloride, telmisartan and glimpiride) have been explored through Bio-MOF-30 for nine, three and three days respectively with its slow release at times. In vitro drugs adsorption studies of Bio-MOF-30 have provided a gateway for in vivo drugs adsorption studies as well for the welfare of humans.

\section{Reference}

1. Li Q, Wu T, Zheng ST, Bu X, Feng P. Single-Walled Polytetrazolate MetalOrganic Channels with High Density of Open Nitrogen-Donor Sites and Gas Uptake. J Am Chem Soc. 2012;134(2):784-787. doi: 10.1021/ ja2092882

2. Li H, Eddaoudi M, OKeeffe M, Yaghi OM. Design and synthesis of an exceptionally stable and highly porous metal-organic framework. Nature. 1999;402:276-279. doi:10.1038/46248

3. Hailian Li, Mohamed Eddaoudi, Jacques Plevert, OKeeffe M, Yaghi OM Ge2ZrO6F2,(H2DAB)H2O:1 A 4- Connected Microporous Material with "Bow Tie" Building Units and an Exceptional Proportion of 3-Rings J Am Chem Soc. 2000;122:12409-12410.

4. Mueller U, Schubert M, Teich F, Puetter H, Schierle-Arndt K, Pastre J. Metal-organic frameworks-prospective industrial applications. J Mater Chem. 2006;16:626-636.

5. James SL. Metal-organic frameworks. Chem Soc Rev. 2003;32:276288

6. Czaja AU, Trukhan N, Meuller U. Industrial applications of metalorganic frameworks. Chem Soc Rev. 2009;38:1284-1293.

7. Tranchemontagne DJ, Mendoza-Cortes JL, O'Keeffe M, Yaghi OM Secondary building units, nets and bonding in the chemistry of metalorganic frameworks. Chem Soc Rev. 2009;38(5):1257-1283. doi: $10.1039 / \mathrm{b} 817735 \mathrm{j}$

8. Long JR, Yaghi OM. The pervasive chemistry of metal-organic frameworks. Chem Soc Rev. 2009;38:1213-1214.

9. Wang Z, Cohen SM. Postsynthetic modification of metal-organic frameworks. Chem Soc Rev. 2009;38:1315-1319.

10.S. M. Cohen, Chem. Sci., 2010, 1, 32-45.

11. Huxford RC, Rocca DJ, Lin W. Metal-organic frameworks as potential drug carriers. Curr Opin Chem Biol. 2010; 14(2):262-268. doi: 10.1016/j.cbpa.2009.12.012
12. Laughreya Z, Gibb BC. Water-soluble, self-assembling container molecules: an update. Chem Soc Rev. 2011;40: 363-386.

13. Wiester MJ, Ulmann PA, Mirkin CA. Enzyme mimics based upon supramolecular coordination chemistry. Angew. Chem. Int. Ed. 2011;50(1):114-137. doi: 10.1002/anie.201000380

14. Amouri H, Desmarets C, Moussa J. Confined Nanospaces in Metallocages: Guest Molecules, Weakly Encapsulated Anions, and Catalyst Sequestration. Chem Rev. 2012;112(4):2015-2041.

15. Chakrabarty R, Mukherjee PS, Stang PJ. Supramolecular coordination: self-assembly of finite two- and three-dimensional ensembles. Chem Rev. 2011;111(11):6810-6918. doi: 10.1021/cr200077m

16. O'Keeffe M, Yaghi OM. Deconstructing the crystal structures of metalorganic frameworks and related materials into their underlying nets. Chem Rev. 2012;112(2):675-702. doi: 10.1021/cr200205j

17. Eubank JF, Wojtas L, Hight MR, Bousquet T, Kravtsov VC, Eddaoudi M. The Next Chapter in MOF Pillaring Strategies: Trigonal Heterofunctional Ligands To Access Targeted High-Connected Three Dimensional Nets, Isoreticular Platforms. Am Chem. Soc. 2011;133:17532-17535.

18. Zheng B, Bai J, Duan J, Wojtas L, Zaworotko MJ. Enhanced $\mathrm{CO}_{2}$ binding affinity of a high-uptake rht-type metal-organic framework decorated with acylamide groups. J Am Chem Soc. 2011;133(4):748-751. doi: 10.1021/ja110042b

19.Zheng B, Yang Z, Bai J, Li Y, Li S. High and selective $\mathrm{CO}_{2}$ capture by two mesoporous acylamide-functionalized rht-type metal-organic frameworks. Chem Commun. 2012;48:7025-7027.

20. Duan J, Yang Z, Bai J, Zheng B, Li Y, Li S. Highly selective CO2 capture of an agw-type metal-organic framework with inserted amides: experimental and theoretical studies. Chem Commun. 2012;48:30583060.

21. Mason JA, Sumida K, Herm ZR, Krishna R, Long JR. Evaluating metalorganic frameworks for post-combustion carbon dioxide capture via temperature swing adsorption. Ener Environ Sci. 2011;4:3030-3039.

22. Sumida K, Rogow DL, Mason JA, McDonald TM, Bloch ED, Herm ZR, et al. Carbon dioxide capture in metal-organic frameworks. Chem Rev. 2011;112(2):724-781. doi: 10.1021/cr2003272

23. Qin JS, Du DY, Li WL, Zhang JP, Li SL, Su ZM, et al. N-rich zeolite-like metal-organic framework with sodalite topology: high $\mathrm{CO}_{2}$ uptake, selective gas adsorption and efficient drug delivery. Chem Sci. 2012;3(6):2114-2118.

24. Vaidhyanathan R, Bradshaw D, Rebilly JN, Barrio JP, Gould JA, Berry NG, et al. A family of nanoporous materials based on an amino acid backbone. Angew. Chem Int Ed. 2006;45(39):6495-6499.

25.An J, Steven JG, Rosi NL. Cation-Triggered Drug Release from a Porous Zinc-Adeninate Metal-Organic Framework. J Am Chem Soc. 2009;131(24):8376-8377. 